\title{
Infeasibility of Systems of Halfspaces
}

\author{
Stefan Felsner and Nicole MoraWe \\ Freie Universität Berlin, Fachbereich Mathematik und Informatik, \\ Takustr. 9, 14195 Berlin, Germany \\ E-mail: \{felsner,morawe\}@inf.fu-berlin.de
}

\begin{abstract}
An oriented hyperplane is a hyperplane with designated good and bad sides. The infeasibility of a cell in an arrangement $\overrightarrow{\mathcal{A}}$ of oriented hyperplanes is the number of hyperplanes with this cell on the bad side. Wit $\operatorname{MinInf}(\overrightarrow{\mathcal{A}})$ we denote the minimum infeasibility of a cell in the arrangement. A subset of hyperplanes of $\overrightarrow{\mathcal{A}}$ is called an infeasible subsystem if every cell in the induced subarrangement has positive infeasibility. With $\operatorname{MaxDis}(\overrightarrow{\mathcal{A}})$ we denote the maximal number of disjoint infeasible subsystems of $\overrightarrow{\mathcal{A}}$. For every arrangement $\overrightarrow{\mathcal{A}}$ of oriented hyperplanes

$$
\operatorname{Min} \operatorname{Inf}(\overrightarrow{\mathcal{A}}) \geq \operatorname{MaxDis}(\overrightarrow{\mathcal{A}}) \text {. }
$$

In this paper we investigate bounds for the ratio of the LHS over the RHS in the above inequality. The main contribution is a detailed discussion of the problem in the case $d=2$, i.e., for 2-dimensional arrangements. We prove that $\operatorname{Min} \operatorname{Inf}(\overrightarrow{\mathcal{A}}) \leq 2 \cdot \operatorname{MaxDis}(\overrightarrow{\mathcal{A}})$, in this case. An example shows that the factor 2 is best possible. If an arrangement $\overrightarrow{\mathcal{A}}$ of $n$ lines contains a cell of infeasibility $n$, then the factor can be improved to $3 / 2$, which is again best possible. We also consider the problem for arrangements of pseudolines in the Euclidean plane and show that the factor of 2 suffices in this more general situation.
\end{abstract}

Mathematics Subject Classifications (2000). 52C30, 52C35, 05 D99.

Key Words. Arrangements, infeasibility, pseudoline, triangular flip.

\section{Introduction}

Let $M x<b$ be a system of $n$ linear inequalities in $d$ variables. Think of $(M, b)$ as an arrangement $\mathcal{A}_{M}$ of affine hyperplanes in $\mathbb{R}^{d}$. From the two halfspaces defined by a hyperplane $\left(m_{i}, x\right)=b_{i}$ we call the halfspace $\left(m_{i}, x\right)<b_{i}$ the good side and the halfspace $\left(m_{i}, x\right)>b_{i}$ the bad side of the hyperplane. The infeasibility of a point $x \in \mathbb{R}^{d}$ is the number of inequalities violated by $x$, i.e., the number of hyperplanes with $x$ on the bad side. We denote the infeasibility of $x$ by $\operatorname{INF}(x)$. Given a cell $c$ in $\mathcal{A}_{M}$ then $\operatorname{InF}(x)=$ $\operatorname{INF}(y)$ for all $x, y \in c$, therefore, we can define the infeasibility of cell $c$ as the infeasibility of any point in $c$. A cell $c$ which is on the good side of all hyperplanes, i.e., $\operatorname{INF}(c)=0$, it is called feasible. An arrangement is called feasible if it contains a feasible cell and infeasible otherwise.

6. Januar 2001 
Let $\overrightarrow{\mathcal{A}}$ be an arrangement of oriented hyperplanes, i.e., of hyperplanes with designated good and bad sides. The cells of minimum infeasibility of $\overrightarrow{\mathcal{A}}$ are of some interest. These cells can be made feasible by removing or reverting the least number of inequalities. We define the minimum infeasibility $\operatorname{MinINF}(\overrightarrow{\mathcal{A}})$ as the minimum of $\operatorname{INF}(c)$ over all cells $c$ of $\overrightarrow{\mathcal{A}}$.

A subarrangement of $\overrightarrow{\mathcal{A}}$ is a subset $\overrightarrow{\mathcal{B}}$ of the hyperplanes of $\overrightarrow{\mathcal{A}}$, with the same orientations. We denote by $\operatorname{MAxDis}(\overrightarrow{\mathcal{A}})$ the maximum number of disjoint infeasible subarrangements of $\overrightarrow{\mathcal{A}}$. Given a cell $c$ of $\overrightarrow{\mathcal{A}}$, a point $x \in c$ and disjoint infeasible subarrangements $\overrightarrow{\mathcal{B}}_{1}, \ldots, \overrightarrow{\mathcal{B}}_{k}$ we note that each $\overrightarrow{\mathcal{B}_{i}}$ contributes at least one to the infeasibility of $x$ in $\overrightarrow{\mathcal{A}}$. From this observation we obtain the following inequality.

Proposition 1. For every arrangement $\overrightarrow{\mathcal{A}}$ of oriented hyperplanes

$$
\operatorname{Min} \operatorname{Inf}(\overrightarrow{\mathcal{A}}) \geq \operatorname{MaxDis}(\overrightarrow{\mathcal{A}}) .
$$

In this paper we investigate bounds for the ratio of the LHS over the RHS in the above inequality. The existence of such a ratio follows from Helly's Theorem, see Proposition 4.

The main contribution of the paper is a detailed discussion of the problem in the case $d=2$, i.e., for 2-dimensional arrangements. We prove that $\operatorname{MinInf}(\overrightarrow{\mathcal{A}}) \leq 2 \cdot \operatorname{MaxDis}(\overrightarrow{\mathcal{A}})$, in this case. An example shows that the factor 2 is best possible. If an arrangement $\overrightarrow{\mathcal{A}}$ of $n$ lines contains a cell of infeasibility $n$, then the factor can be improved to $3 / 2$, which is again best possible. Finally, we consider the problem for arrangements of pseudolines in the Euclidean plane. A completely independent proof is required to show that the factor of 2 suffices in this more general situation.

The problem of investigating the gap in Proposition 1 came to our attention through a question posed by Komei Fukuda at the Monte Verità Conference on Discrete and Computational Geometry 1998. Fukuda remarked that he was led to the problem by the observation that some well studied graph problems can be viewed as problems about minimum infeasibility of certain arrangements. We briefly discuss two such instances.

The Maximum Acyclic Subgraph problem asks for the minimum number of arcs of a directed graph $D=(V, A)$ whose removal makes the graph acyclic (see e.g. [7]). Associate a variable $x_{v}$ with vertex $v$ and an inequality $x_{u}-x_{v} \geq 1$ with each arc $(u, v)$. The Maximum Acyclic Subgraph problem is the problem of determining $\operatorname{INF}(\overrightarrow{\mathcal{A}})$ for the corresponding arrangement. A minimal infeasible subarrangement corresponds to a simple cycle, hence, Proposition 1 translates to the lower bound given by a maximum collection of disjoint cycles. 
The Lucchesi-Younger Theorem for directed graphs is a MinMax result which can be interpreted as a situation in which equality in Proposition 1 holds (for proofs see $[10,9,5]$ ). Let $D=(V, A)$ be a directed graph with a 2-connected underlying graph $G_{D}$. A dicut of $D$ is a set $B$ of arcs which is a cut $(X, Y)$ for $G_{D}$ such that all arcs of $B$ are oriented from $X$ to $Y$. The Lucchesi-Younger Theorem states that the minimum number of arcs of $D$ that have to be reversed to make $D$ strongly connected equals the maximum number of disjoint dicuts of $D$. For the translation provide a variable $x_{a}$ for each arc of $D$, now consider the flow constraint for each vertex $v \in V$, i.e., $\sum_{a \in i n(v)} x_{a}-\sum_{a \in o u t(v)} x_{a}=0$, and positivity inequalities $x_{a}>0$. The theorem is equivalent to $\operatorname{Min} \operatorname{Inf}(\overrightarrow{\mathcal{A}})=\operatorname{MaxDis}(\overrightarrow{\mathcal{A}})$ when $\overrightarrow{\mathcal{A}}$ is the arrangement induced by the inequalities on the $(|A|-|V|)$-dimensional affine space defined by the flow constraints.

\section{Introductory Observations and Examples}

In this section we collect some general observations about infeasibility in arrangements of oriented hyperplanes. Without proof we state the following two observations.

Observation 2. An infeasible arrangement in $\mathbb{R}^{d}$ contains at least $d+1$ hyperplanes.

For the sake of simplicity we assume throughout the paper that the arrangements we consider are 'in general position'. By this we mean that there are no parallel hyperplanes and no point is contained in more than $d$ of the hyperplanes of the arrangement. Our results hold without this assumption, but the proofs would be broadened by additional details.

Observation 3. An arrangement in $\mathbb{R}^{d}$ consisting of $d+1$ hyperplanes is infeasible iff its (unique) bounded cell has infeasibility $d+1$.

An infeasible arrangement in $\mathbb{R}^{d}$ consisting of $d+1$ hyperplanes is called a infeasible base arrangement.

Lemma 1. Every infeasible arrangement contains an infeasible base arrangement.

Proof. Let $\overrightarrow{\mathcal{A}}$ be an infeasible arrangement. Suppose that $\overrightarrow{\mathcal{A}}$ contains no infeasible base arrangement. This means that for each set of $d+1 \operatorname{good}$ halfspaces there is a point being contained in all of them. Helly's Theorem 
implies the existence of a point $x$ which is contained in all the good halfspaces. By definition this point and, hence, his cell has infeasibility 0 . This means that $\overrightarrow{\mathcal{A}}$ is feasible, contradiction.

Proposition 4. For every arrangement $\overrightarrow{\mathcal{A}}$ in $\mathbb{R}^{d}$

$$
\operatorname{MinInf}(\overrightarrow{\mathcal{A}}) \leq(d+1) \cdot \operatorname{MaxDis}(\overrightarrow{\mathcal{A}})
$$

Proof. Recursively remove infeasible base arrangements from $\overrightarrow{\mathcal{A}}$ until the arrangement becomes feasible. This is possible by Lemma 1 . Let $x$ be a point in the feasible cell. The removal of each infeasible base arrangements has decreased the infeasibility of $x$ by at most $d+1$. Therefore, $\operatorname{Min} \operatorname{InF}(\overrightarrow{\mathcal{A}}) \leq$ $\operatorname{MinInF}(x) \leq(d+1) \cdot \#$ removed subarrangements $\leq(d+1) \cdot \operatorname{MaxDis}(\overrightarrow{\mathcal{A}})$.

We turn to a lower bound construction. Choose $n$ points uniformly at random from the unit sphere in $\mathbb{R}^{d}$. With each point consider the tangent hyperplane and let the bad halfspace of the hyperplane be the side containing the sphere. The infeasibility of a point $x$ in the interior of the sphere is $n$. Along every ray starting at $x$ the infeasibility is monotonically decreasing. Therefore, every cell of minimum infeasibility is an unbounded cell. The infeasibility of an unbounded cell of the arrangement equals the number of random points on the averted hemisphere. By construction this number will be approximately $n / 2$. Since a infeasible subsystem consists of $d+1$ halfspaces, there are at most $n /(d+1)$ of them. On the basis of this idea there is a rigorous proof for the next proposition.

Proposition 5. For every $\epsilon>0$ there are arrangements $\overrightarrow{\mathcal{A}}$ in $\mathbb{R}^{d}$ with

$$
\operatorname{Min} \operatorname{Inf}(\overrightarrow{\mathcal{A}}) \geq\left(\frac{d+1}{2}-\epsilon\right) \cdot \operatorname{MaxDis}(\overrightarrow{\mathcal{A}}) .
$$

As a warmup we consider the very simple case of 1-dimensional arrangements. A hyperplane in dimension $d=1$ is just a point $p$ in $\mathbb{R}$ and its halfspaces are the two open intervals ] $-\infty, p$ [ and ] $p, \infty[$. A cell of an arrangement corresponds to an (open) interval. An infeasible base arrangement consists of two points with disjoint good halfspaces, see Figure* 1 .

Let $\overrightarrow{\mathcal{A}}$ be a 1-dimensional arrangement. Since we are only interested in the infeasibility of the cells of $\overrightarrow{\mathcal{A}}$, we disregard the exact position of the points (hyperplanes) and restrict the attention to their ordering and the

\footnotetext{
*In figures we mark the good side of a hyperplane with a flag.
} 


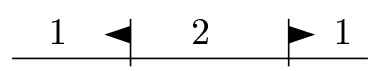

Figure 1: An infeasible base arrangement in one dimension, cells are labeled with their infeasibility.

orientation information, i.e., whether the left or the right side is the good one. From $\overrightarrow{\mathcal{A}}$ construct a $\{+,-\}$-sequence containing $n$ signs: Scan the points from left to right. If the bad side is to the right of the point write a plus-sign, otherwise, write a minus-sign. Infeasible base arrangements of $\overrightarrow{\mathcal{A}}$ are in one-to-one correspondence to the +- subsequences of this sequence. A +- subsequence of consecutive signs will be called tight subarrangement.

Observation 6. A 1-dimensional infeasible arrangement contains a tight subarrangement.

Observation 7. The bounded cell of a tight subarrangement of $\overrightarrow{\mathcal{A}}$ is not a cell of minimal infeasibility in $\overrightarrow{\mathcal{A}}$.

Observation 7 is proven by considering the infeasibility of one of the two cells adjacent to the cell between the points of the tight subarrangement. From this observation it follows that removing a tight subarrangement from $\overrightarrow{\mathcal{A}}$ decreases the infeasibility of the arrangement by one.

Given an infeasible arrangement $\overrightarrow{\mathcal{A}}$, recursively remove tight subarrangements, as long as possible. When the procedure stops the arrangement is feasible (Observation 6). This shows

Proposition 8. For every 1-dimensional arrangement $\overrightarrow{\mathcal{A}}$

$$
\operatorname{Min} \operatorname{Inf}(\overrightarrow{\mathcal{A}})=\operatorname{MaxDis}(\overrightarrow{\mathcal{A}}) .
$$

\section{Arrangements of Lines}

In this section we discuss arrangements of lines in 2-dimensional space. With every line we assume that a good and a bad halfplane are specified. An infeasible base arrangement is shown in Figure 2.

It is easy to give a more explicit example for the lower bound of Proposition 5 in dimension two. Consider the arrangement of lines supported by the edges of a regular $n$-gon with orientations such that all halfspaces containing the $n$-gon are bad. In the even case small perturbations are required to avoid parallel lines. It is easy to see that $\operatorname{MaxDis}(\overrightarrow{\mathcal{A}})=\lfloor n / 3\rfloor$ and $\operatorname{MinInF}(\overrightarrow{\mathcal{A}})=\lfloor(n-1) / 2\rfloor$. An example with $n=5$ is shown in Figure 3 .

The main result of this section is the following theorem. 


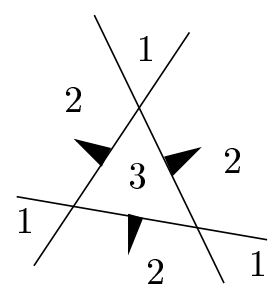

Figure 2: An infeasible base arrangement in $\mathbb{R}^{2}$, cells are labeled with their infeasibility.

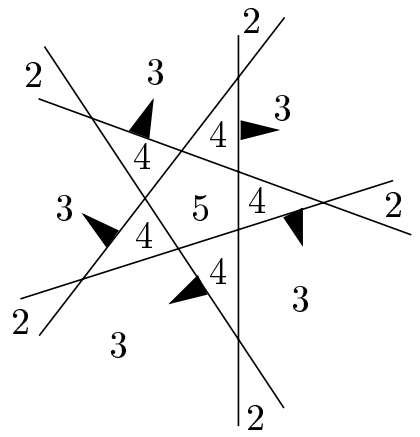

Figure 3: Arrangement with $\operatorname{MaxDis}(\overrightarrow{\mathcal{A}})=1$ and $\operatorname{Min} \operatorname{Inf}(\overrightarrow{\mathcal{A}})=2$

Theorem 9. For every arrangement $\overrightarrow{\mathcal{A}}$ of oriented lines

$$
\operatorname{Min} \operatorname{Inf}(\overrightarrow{\mathcal{A}}) \leq 2 \cdot \operatorname{MaxDis}(\overrightarrow{\mathcal{A}}) .
$$

This bound is best possible.

There is a class of arrangements which allows to prove a smaller factor.

Theorem 10. For every arrangement $\overrightarrow{\mathcal{A}}$ of oriented lines with a cell which is on the bad side of every line

$$
\operatorname{Min} \operatorname{Inf}(\overrightarrow{\mathcal{A}}) \leq\lceil(3 / 2) \cdot \operatorname{MaxDis}(\overrightarrow{\mathcal{A}})\rceil
$$

This bound is best possible.

\subsection{Proof of Theorem 9}

The proof is based on a recursive removal of infeasible base arrangements. The goal is to find a subarrangement whose removal decreases the infeasibility of the remaining arrangement by at most two, we call such a infeasible base subarrangement a tight subarrangement. 
For a cell $c$ of minimum infeasibility let $\mathcal{A}(c)$ be the arrangement formed by all lines having $c$ on their bad side. Let $z(c)$ be the cell in the arrangement $\mathcal{A}(c)$ containing $c$. With $d_{c}$ we denote be the minimal distance between a point from cell $c$ (including its boundary) and a line of $\overrightarrow{\mathcal{A}}(c)$. The minimal distance $d_{c}$ is greater than 0 , since the boundary of $c$ is not contained in $\overrightarrow{\mathcal{A}}(c)$. Note that $d_{c}$ is achieved at a corner of $c$. Among all cells of minimum infeasibility choose the cell $c$ which minimizes $d_{c}$. Let $p$ be the corner of $c$ and $l$ be the line of $\mathcal{A}(c)$ such that $d_{c}=\operatorname{dist}(p, l)$. With the following two lemmas we show that line $l$ and the two lines $l_{1}, l_{2}$ crossing at $p$ form a tight subarrangement. Figure 4 illustrates the construction.

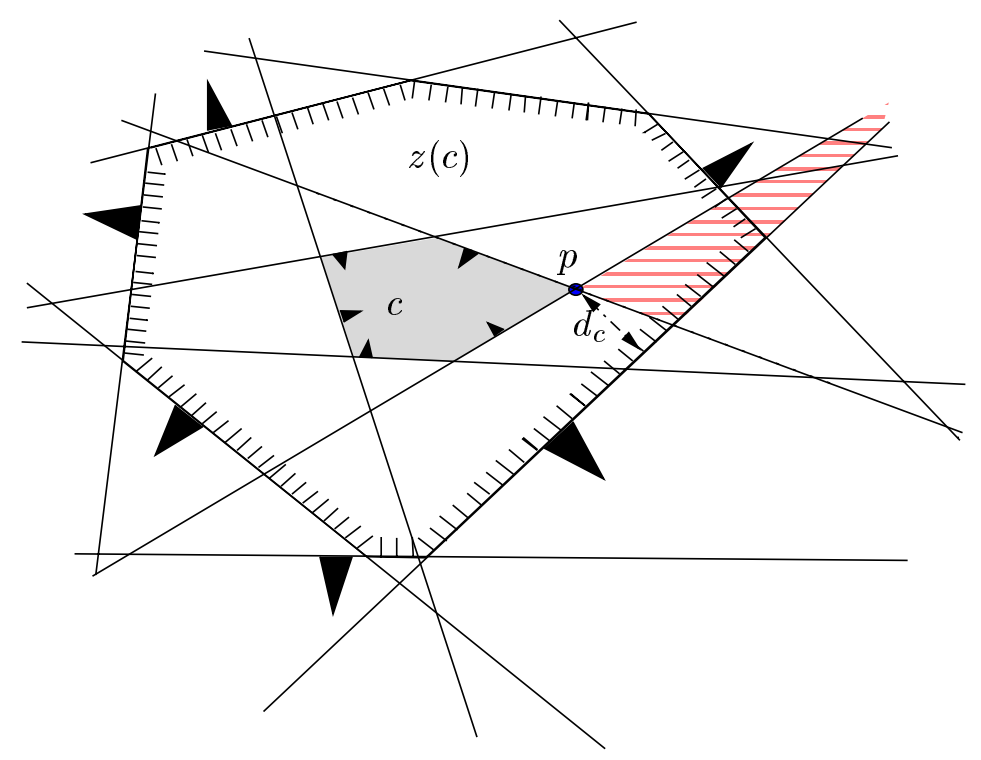

Figure 4: The construction: The large 7-gon is $z(c)$ and the fasciated region is the triangle of the subarrangement $l, l_{1}, l_{2}$.

Lemma 2. The subarrangement $l, l_{1}, l_{2}$ is infeasible.

Proof. Lines $l_{1}$ and $l_{2}$ divide the plane into four open unbounded cells as shown in Figure 5 . If $l$ crosses region $R$ the subsystem formed by the three lines is clearly infeasible. This follows since otherwise $c$ would contain points which are closer to $l$ than $p$, in contradiction to the definition of $d_{c}$.

Lemma 3. Removing the subarrangement $l, l_{1}, l_{2}$ decreases the infeasibility of the remaining arrangement by at most two. 


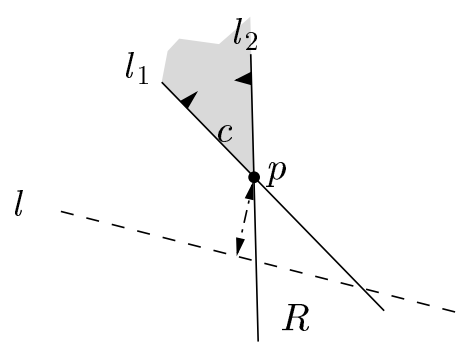

Figure 5: The relative positions of $l_{1}, l_{2}$ and $l$.

Proof. We have to show that there is no cell in the triangular cell formed by $l, l_{1}, l_{2}$ which is of minimal infeasibility.

Suppose $\bar{c}$ is a cell with minimal infeasibility contained in the triangular region of $l, l_{1}, l_{2}$. Define $\overrightarrow{\mathcal{A}}(\bar{c})$ as above. Then $l$ belongs to $\overrightarrow{\mathcal{A}}(\bar{c})$ and we get $d_{\bar{c}} \leq \operatorname{dist}(\bar{c}, l)<d_{c}$. Since we have chosen $c$ as the cell of minimal infeasibility minimizing $d_{c}$, the existence of such a cell $\bar{c}$ is impossible.

Since removing a tight subarrangement decreases the infeasibility of every cell of minimal infeasibility by at most two we get $\operatorname{Min} \operatorname{Inf}(\overrightarrow{\mathcal{A}}) \leq$ $2 \cdot \#$ removed subarrangements $\leq 2 \cdot \operatorname{MAxDis}(\overrightarrow{\mathcal{A}})$. This completes the proof of the inequality of Theorem 9 .

To complete the proof of Theorem 9 we need a construction showing that the factor 2 is best possible. Such a construction will be given in Subsection 4.2 .

Remark. The idea of this proof generalizes to higher dimensions. An analogous distance argument allows to detect an infeasible base subarrangement whose removal decreases the infeasibility of every minimal infeasible cell by at most $d$. This improves the factor of Proposition 4 and gives: For every arrangement $\overrightarrow{\mathcal{A}}$ in $\mathbb{R}^{d}$

$$
\operatorname{Min} \operatorname{Inf}(\overrightarrow{\mathcal{A}}) \leq d \cdot \operatorname{MaxDis}(\overrightarrow{\mathcal{A}}) .
$$

\subsection{Proof of Theorem 10}

Recall that now we deal with line-arrangements with one cell being on the bad side of every line. In this case all cells of minimal infeasibility are unbounded and we restrict the attention to those cells which intersect the circle at infinity.

We encode the infeasibility of all unbounded cells with a $\{+1,-1\}$ sequence of length $n$ : Let $k$ be the minimum infeasibility of the arrangement 
and let $x$ be a point with infeasibility $k$ on the circle at infinity. Let $y$ be the antipodal point of $x$. Note that the infeasibility of $y$ is $n-k$. Traversing the lines at infinity clockwise from $x$ to $y$ we write +1 , if $x$ is on the good side of a line, and we write -1 if $x$ is on the bad side.

The infeasibility of every unbounded cell $c$ is encoded by this sequence. Let the unbounded cells be numbered $0,1, \ldots, 2 n-1$ in clockwise order, such that cell 0 contains $x$. If $c$ is the $i$-th cell with $i \leq n$, then the infeasibility of $c$ is $k$ plus the sum of the first $i$ numbers of the sequence. Otherwise, if $i>n$, use its antipodal cell $c^{*}$ to calculate the infeasibility as $\operatorname{INF}(c)=n-\operatorname{INF}\left(c^{*}\right)$.

By the choice of $x$ as a point of minimal infeasibility (and thereby $y$ as a point of maximal infeasibility) the resulting sequence has a total sum of $n-2 k$ and the property that every prefix and postfix has a non-negative sum.

The next lemma describes how the sequence represents infeasible base subarrangements. We abbreviate +1 and -1 with + and - .

Lemma 4. Infeasible base arrangements are represented in the,+- sequence by +-+ or -+- subsequences.

Proof. Fix an infeasible base arrangement. Then $x$ is either contained in one of its regions with infeasibility 1 or infeasibility 2. Constructing the sequences transforms the base arrangement to +-+ in case of $x$ lying in a region of infeasibility 1 and -+- otherwise.

Conversely, let +-+ be a subsequence of the sequence. With respect to $x$ there are only two possibilities for the relative position of its three lines. The induced orientation of +-+ leads to the two configurations show in Figure 6 . The left configurations in fact is an infeasible base arrangement. The right configuration is not possible, since no point lies in all three bad halfplanes. The same reasoning applies to -+- subsequences.

To complete the proof of Theorem 10 we need a combinatorial lemma about decompositions of sequences of plus- and minus-signs.

Lemma 5. Let $S$ be a $\{+,-\}$-sequence containing $n-k$ plus-signs and $k$ minus-signs with the property that every prefix and postfix of $S$ has a nonnegative sum. Then $S$ contains at least $\left\lfloor\frac{2 k+1}{3}\right\rfloor$ disjoint subsequences of the form +-+ or -+- .

Proof. We abstract the string $S$ to a a zig-zag path with steps $(1,1)$ and $(1,-1)$ starting at $(0, k)$ and ending at $(n, n-k)$, always staying in the $y$-interval $[k, n-k]$, see Figure 7 . 

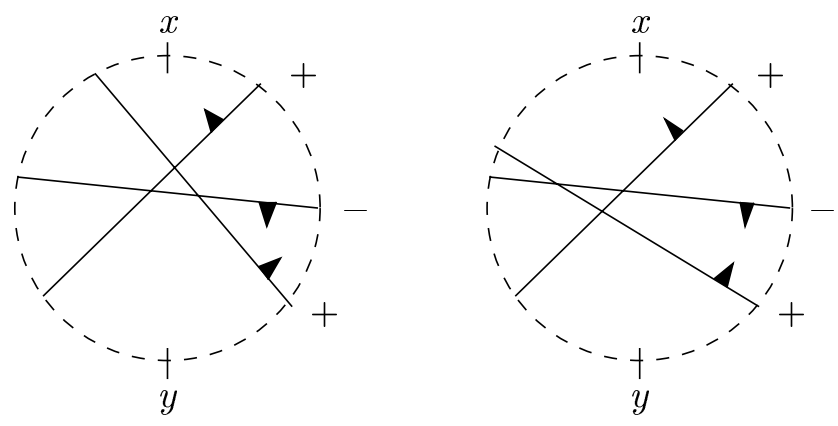

Figure 6: The two arrangements defined by +-+ .

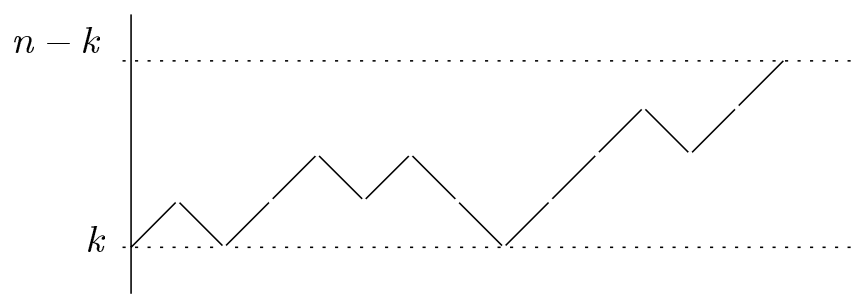

Figure 7: The zig-zag path for the string +-++-+--+++-++ .

A level of $S$ is the set of symbols corresponding to a step in the zig-zag path with $y$-coordinates between $i$ and $i+1$, for some $i$. Note that each level contains either zero or an odd number of symbols.

We prove the lemma by induction on the length of the string. If the string contains only one symbol it is + and thus

$$
\text { \#disjoint alternating triples }=0=\left\lfloor\frac{2 \cdot 0+1}{3}\right\rfloor .
$$

We distinguish several reducing operations on the sequence.

1. There is a level which contains exactly one symbol:

This symbol must be + . Removing it leads to a shorter valid string with the same number of negative entries. By induction this shorter sequence contains $\left\lfloor\frac{2 k+1}{3}\right\rfloor$ disjoint alternating triples.

2. There is a level which contains exactly three symbols:

These three symbols form the substring +-+ . Removing them leaves a shorter valid string with $k-1$ negative symbols. By induction

$$
\text { \#disjoint alternating triples } \geq\left\lfloor\frac{2(k-1)+1}{3}\right\rfloor+1 \geq\left\lfloor\frac{2 k+1}{3}\right\rfloor \text {. }
$$


3. There is a level containing at least six symbols:

The first six symbols of this level form the substring +-+-+- , which consists of two alternating triples. Removing all six symbols leaves a shorter valid string with $k-3$ negative symbols. Thus

$$
\text { \#disjoint alternating triples } \geq\left\lfloor\frac{2(k-3)+1}{3}\right\rfloor+2=\left\lfloor\frac{2 k+1}{3}\right\rfloor .
$$

4. If non of the above cases holds, then every level contains exactly five symbols.

- If there is only one level, then $n=5, k=2$ and the first three symbols form a substring +-+ . Hence,

$$
\text { \# disjoint alternating triples }=1=\left\lfloor\frac{2 \cdot 2+1}{3}\right\rfloor .
$$

- If there are at least two levels, then consider the 10 symbols of height $k$ and $k+1$. This substring has the shape of one of the 6 cases shown in Figure 8. The figure indicates how the first nine symbols of each string can be decomposed into three disjoint alternating triples. Removing the ten symbols leaves a valid string with $k-4$ negative symbols. Thus

$$
\text { \#disjoint alternating triples } \geq\left\lfloor\frac{2(k-4)+1}{3}\right\rfloor+3 \geq\left\lfloor\frac{2 k+1}{3}\right\rfloor .
$$

Since, by construction the parameter $k$ was the infeasibility of the arrangement, the lemma gives $\operatorname{MaxDis}(\overrightarrow{\mathcal{A}}) \geq\left\lfloor\frac{2}{3} \operatorname{Min} \operatorname{Inf}(\overrightarrow{\mathcal{A}})+\frac{1}{3}\right\rfloor$. This implies $\left\lceil\frac{3}{2} \operatorname{MaxDis}(\overrightarrow{\mathcal{A}})\right\rceil \geq \operatorname{MinInF}(\overrightarrow{\mathcal{A}})$, i.e., the formula stated in the theorem.

The tightness of the factor $3 / 2$ is shown via the arrangement of the $n$ gon. Consider the arrangement of lines supported by the edges of a regular $(6 r+5)$-gon oriented so that all halfspaces containing the polygon are bad. Then $\operatorname{MaxDis}(\overrightarrow{\mathcal{A}})=2 r+1$ and $\operatorname{MinINF}=3 r+2=\left\lceil\frac{3}{2} \operatorname{MaxDis}(\overrightarrow{\mathcal{A}})\right\rceil$.

\section{Arrangements of Pseudolines}

A pseudoline is a curve in $\mathbb{R}^{2}$ whose removal from the plane leaves two unbounded connected components. In other words a pseudoline is a simple 

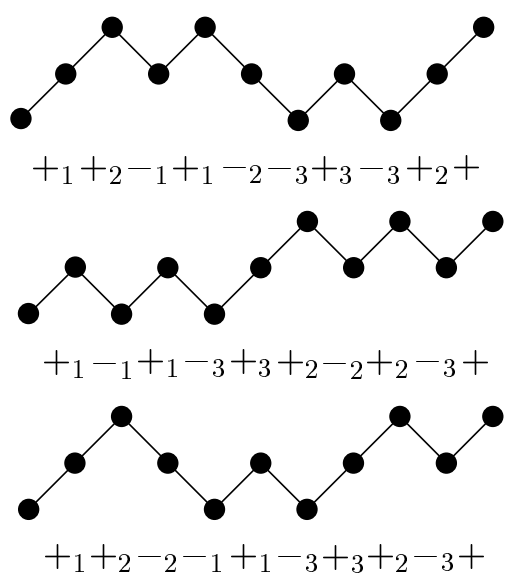
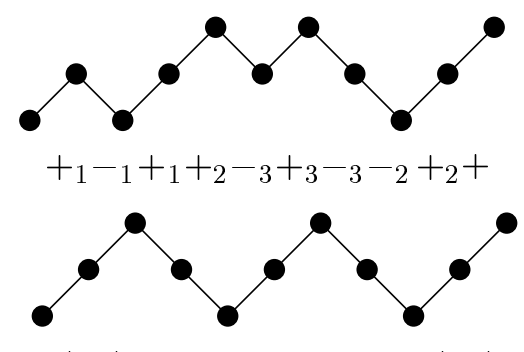

$+{ }_{1}+{ }_{2}-{ }_{3}-{ }_{1}+{ }_{1}+{ }_{3}-{ }_{3}-{ }_{2}+{ }_{2}+$

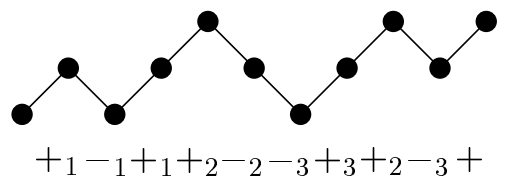

Figure 8: The six possible patterns of two levels, each of 5 symbols

curve which goes to infinity on both sides. An arrangement of pseudolines is a family of pseudolines with the property that each pair of pseudolines has a unique point of intersection, where the two pseudolines cross. Arrangements of pseudolines are a natural generalization of arrangements of straight lines, they have been studied in a wide variety of contexts. Grünbaum [8] is a nice little monograph collecting results and many problems about arrangements of both straight-lines and pseudolines. A more recent overview is given by Goodman [6].

Let $\mathcal{A}$ be an arrangement of pseudolines. Choose an orientation for each pseudoline, or equivalently, assign a good and a bad side to each pseudoline. For the oriented arrangement $\overrightarrow{\mathcal{A}}$ there are natural notions of infeasibility for points, cells and subarrangements. Hence, questions regarding the relation between $\operatorname{Min} \operatorname{Inf}(\overrightarrow{\mathcal{A}})$ and $\operatorname{MaxDis}(\overrightarrow{\mathcal{A}})$ can be asked in this more general setting. Let us review the results for 2-dimensional arrangements of lines and see what can be adapted to pseudolines. The validity of Propositions 1 in the new context is obvious. The upper bound of Proposition 4, i.e., $\operatorname{MinInf}(\overrightarrow{\mathcal{A}}) \leq 3 \cdot \operatorname{MaxDis}(\overrightarrow{\mathcal{A}})$, holds, because of an analog of Helly's Theorem for pseudo-halfspaces. What about pseudoline analogs of Theorems 9 and 10? In the case of Theorem 10 the answer is easy. The proof given in Subsection 3.2 is completely combinatorial, it makes no use of straightness. Therefore, the result remains valid in the setting of arrangements of pseudolines:

Theorem 11. For every arrangement $\overrightarrow{\mathcal{A}}$ of oriented pseudolines with a cell 
which is on the bad side of every pseudoline

$$
\operatorname{Min} \operatorname{Inf}(\overrightarrow{\mathcal{A}}) \leq\lceil(3 / 2) \cdot \operatorname{MaxDis}(\overrightarrow{\mathcal{A}})\rceil
$$

This bound is best possible.

The proof of Theorem 9 in Subsection 3.1 makes use of a metric argument. Therefore, the question whether the factor of 2 remains valid for arrangements of pseudolines cannot be answered on the basis of the old proof. The main result of this section is a proof of the following generalization of Theorem 9.

Theorem 12. For every arrangement $\overrightarrow{\mathcal{A}}$ of oriented pseudolines

$$
\operatorname{Min} \operatorname{Inf}(\overrightarrow{\mathcal{A}}) \leq 2 \cdot \operatorname{MaxDis}(\overrightarrow{\mathcal{A}})
$$

This bound is best possible.

In the next subsection we collect preparing facts about arrangements of pseudolines. Along the way we learn some combinatorics of cyclic arrangements and use this to produce the lower bound example for Theorem 9 . Subsection 4.3 contains the main body of the proof for Theorem 12 .

\subsection{Basic Facts}

Given an arrangement $\mathcal{A}$ of pseudolines, choose a unbounded cell $\hat{c}$ and imagine that $\hat{c}$ contains the northpole. The complementary cell $\check{c}$ is the unbounded cell separated from $\hat{c}$ by all the pseudolines of the arrangement. Label the pseudolines so that traversing the circle at infinity counterclockwise from $\hat{c}$ to $\check{c}$ they are met in the order $1,2, \ldots, n$. This results in a marked arrangement of pseudolines.

On the set of all (combinatorially different) marked arrangements of $n$ pseudolines consider a graph $\mathcal{G}_{n}$ whose edges correspond to triangular flips. To be precise, the pair $(\overrightarrow{\mathcal{A}}, \overrightarrow{\mathcal{B}})$ is an edge of $\mathcal{G}_{n}$ iff there are three pseudolines $i<j<k$ in $\overrightarrow{\mathcal{A}}$ such that:

- There is a triangular cell bounded by the tree pseudolines $i, j, k$.

- The northpole is separated from the crossing of pseudolines $i$ and $k$ by pseudoline $j$.

- Arrangement $\overrightarrow{\mathcal{B}}$ is equivalent to the arrangement obtained from $\overrightarrow{\mathcal{A}}$ by pulling pseudoline $j$ below the crossing of $i$ and $k$.

Figure 9 shows an example. 


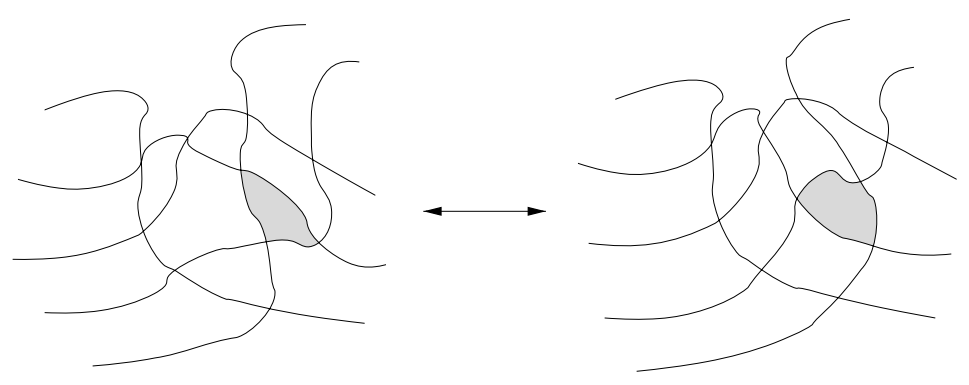

Figure 9: A flip at the shaded triangular cell.

Various aspects of the graph $\mathcal{G}_{n}$ have been studied in $[3,2,1,4]$. We will need the following properties of $\mathcal{G}_{n}$.

(1) There is a unique arrangement $\mathcal{C}$ of $n$ pseudolines, so that the indegree of $\mathcal{C}$ in $\mathcal{G}_{n}$ is zero. The arrangement $\mathcal{C}$ is the cyclic arrangement, it will be studied in more detail in the next subsection.

(2) $\mathcal{G}_{n}$ is the diagram of a ranked poset. (This poset is the "higher Bruhat order" $B(n, 2)$ introduced in [11] and further studied in [12].)

The general idea for the proof of Theorem 12 is as follows. In a first step we show the factor of 2 for all orientations of the cyclic arrangement. This is done in Subsection 4.2. To prove the same factor for a marked oriented arrangement $\overrightarrow{\mathcal{A}}$ consider a path $\mathcal{C}=\overrightarrow{\mathcal{A}_{0}}, \overrightarrow{\mathcal{A}_{1}}, \ldots, \overrightarrow{\mathcal{A}_{r}}=\overrightarrow{\mathcal{A}}$ in $\mathcal{G}_{n}$. Let $\overrightarrow{\mathcal{A}_{i}}$ be the orientation of $\mathcal{A}_{i}$ where line $j$ is oriented as in $\mathcal{A} 0$. The main step is to show that $\operatorname{Min} \operatorname{Inf}\left(\overrightarrow{\mathcal{A}}_{i}\right) \leq 2 \cdot \operatorname{MaxDis}\left(\overrightarrow{\mathcal{A}}_{i}\right) \operatorname{implies} \operatorname{MinInf}\left(\overrightarrow{\mathcal{A}}_{i+1}\right) \leq$ $2 \cdot \operatorname{MaxDis}\left(\overrightarrow{\mathcal{A}}_{i+1}\right)$. Actually the proof given in Subsection 4.3 is a bit more complicated.

\subsection{Cyclic Arrangements}

The marked cyclic arrangement $\mathcal{C}$ of $n$ lines is characterized by the property that for any three lines $i<j<k$ the crossing of $i$ and $k$ is separated from the northpole by line $j$. A straight-line realization of this arrangement can be obtained by choosing $n$ different tangents to the parabola $y=x^{2}$, the special north-cell $\hat{c}$ is the cell containing the parabola. Figures 11 and 12 show cyclic arrangements.

An orientation of the cyclic arrangement, i.e., an assignment of good and bad sides to all lines, can be encoded by a sequence $S$ of + and - signs. Take the lines in the order of their labels and write a + if the northern side 
is bad and - if the northern side is good. Figure 10 illustrates the following observation.

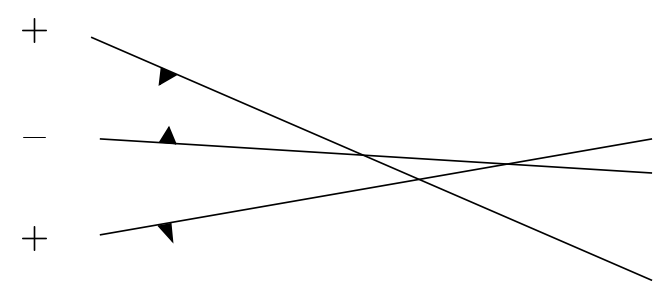

Figure 10: Infeasible base arrangement in a cyclic arrangement.

Observation 13. Let $\overrightarrow{\mathcal{C}}$ be an oriented cyclic arrangement. Infeasible base arrangements of $\overrightarrow{\mathcal{C}}$ correspond one-to-one to the +-+ subsequences in the sequence $S$ of the orientation.

Given the sequence $S$ of an orientation of $\overrightarrow{\mathcal{C}}$ we want to calculate the infeasibility of a cell $c$. The infeasibility of the north-cell $\hat{c}$ equals the number of plus-signs in $S$. The infeasibility of a cell $c$ different from the north-cell is obtained by reverting in $S$ the signs of all the lines above $c$ and then counting the number of plus-signs.

Associate with every cell $c$ of the cyclic arrangement $\mathcal{C}$ of $n$ lines associate the set of lines above $c$, i.e., the set of those lines separating $c$ from the northpole. This gives a bijective mapping from the cells of $\overrightarrow{\mathcal{C}}$ to intervals $[i, \ldots, j]$ with $1 \leq i \leq j \leq n$, together with $\emptyset$. Figure 11 exemplifies the correspondence.

We are ready, now, to provide the lower bound example for Theorems 9 and 12 .

Proposition 14. There is an orientation $\overrightarrow{\mathcal{C}}$ of the cyclic arrangement $\mathcal{C}$ with $4 k+1$ lines, such that $\operatorname{MaxDis}(\overrightarrow{\mathcal{C}})=k$ and $\operatorname{Min} \operatorname{Inf}(\overrightarrow{\mathcal{C}})=2 k$.

Proof. Consider the orientation $\overrightarrow{\mathcal{C}}$ of the cyclic arrangement corresponding to the sequence $+[-+-+]^{k}$ of length $4 k+1$. Figure 12 shows an example with $k=2$.

There are only $2 k+1$ plus-signs in the sequence and every +-+ subsequence requires two, therefore, there can be at most $k$ disjoint +-+ subsequences. Obviously, there are as many and $\operatorname{MaxDis}(\overrightarrow{\mathcal{C}})=k$.

Reverting the signs in an interval of $S$ can decrease the number of plussigns by at most one. Therefore, the infeasibility of every cell in that arrangement is at least $2 k$. 


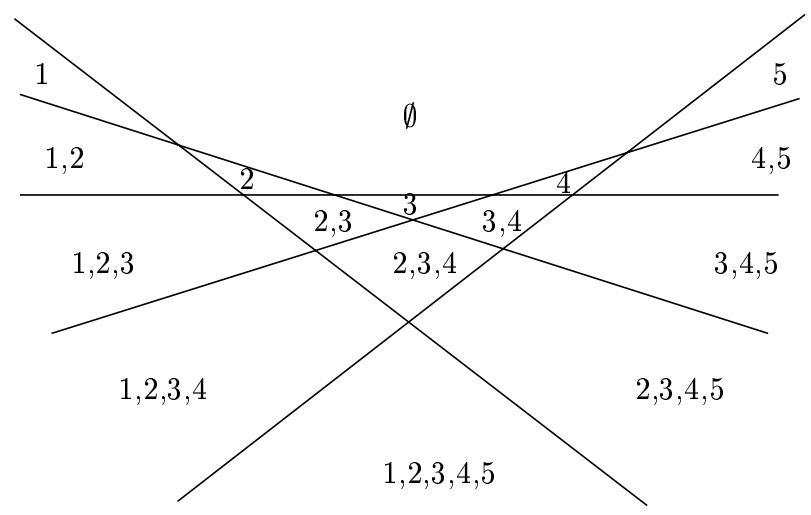

Figure 11: Cyclic arrangement with $n=5$, showing the labels of lines above cells.

Cyclic arrangements can be realized as straight-line arrangements, therefore, the statement of the next proposition is a consequence of Theorem 9 . Here we give a new proof which has the virtue of being purely combinatorial.

Proposition 15. Given an orientation $\overrightarrow{\mathcal{C}}$ of the cyclic arrangement with $\operatorname{MaxDis}(\overrightarrow{\mathcal{C}})=k$, then there is a cell $c$ with $\operatorname{INF}(c) \leq 2 k$.

Proof. The orientation $\overrightarrow{\mathcal{C}}$ is encoded by a sequence $S$ with no more then $k$ disjoint +-+ subsequences. We have to show that there is an interval such that reverting the signs of this interval results in a sequence with at most $2 k$ plus-signs.

Define the span of a subset $T$ of signs of $S$ as the length of the shortest interval containing all the signs of $T$. The span a family of $k$ disjoint +-+ subsequences of $S$ is the sum of the spans of its $k$ triples.

Let $F$ be a family of $k$ disjoint +-+ triples of $S$ such that the span of $F$ if minimal among all such families. In $S$ color the $3 k$ signs from the triples in $F$ red and all the remaining signs blue. Since, the blue signs are not allowed to contain an additional +-+ subsequence, the induced blue sequence is described by the regular expression $[-]^{*}[+]^{*}[-]^{*}$.

For the reversal we choose the smallest interval containing all the blue plus-signs. Let $S^{\prime}$ be the sequence after reversal of the signs in this interval. In $S^{\prime}$ there is no blue plus-sign. Let $T$ be one of the $k$ red +-+ triples of $F$ and let $T^{\prime}$ be the corresponding triple in $S^{\prime}$. We claim that $T^{\prime}$ contains at most two plus-signs. Since all plus-signs of $S^{\prime}$ are red the claim immediately yields an upper bound of $2 k$ for the number of plus-signs in $S^{\prime}$, which proves the proposition. 


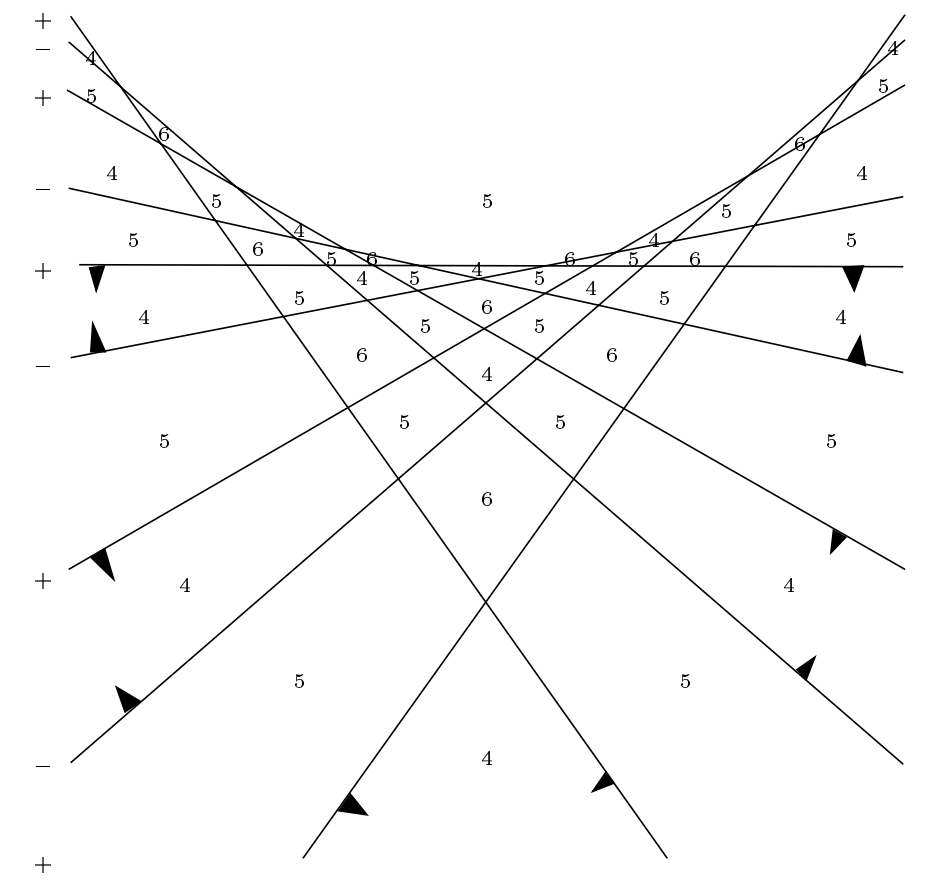

Figure 12: Cyclic arrangement with $\operatorname{MaxDis}(\overrightarrow{\mathcal{C}})=2$ and $\operatorname{MinInf}(\overrightarrow{\mathcal{C}})=4$.

Suppose $T$ is a triple in $F$ violating the claim. This means, that the middle symbol, the -, of $T$ has been reversed, while the other two symbols remained unchanged. By the choice of the interval for reversal we find that the first and the last blue plus-sign together with the - from $T$ form a +-+ subsequence $T^{*}$ in $S$. The span of $T^{*}$ is less then the span of $T$. Therefore, $F-T+T^{*}$ is a family of $k$ disjoint +-+ subsequences and span less then the span of $F$, contradiction.

Remark. The notion of a cyclic arrangement is not restricted to the plane. Let $\mathcal{C}_{o}$ be the orientation of such an arrangement given by alternating sequence of plus- and minus-signs. In even dimension $d$ it can be shown that $\operatorname{Min} \operatorname{Inf}(\overrightarrow{\mathcal{C}}) \geq \frac{d+2}{2} \operatorname{MaxDis}(\overrightarrow{\mathcal{C}})-\frac{d}{2}$. This is a slight improvement over the lower bound of Proposition 5. In odd dimensions the gain is smaller. Compared to Proposition $5 \overrightarrow{\mathcal{C}}$ has the advantage of providing a deterministic construction for the factor $(d+1) / 2$ and saving the $\epsilon$. 


\subsection{Proof of Theorem 12}

Let $\overrightarrow{\mathcal{A}}$ be an oriented arrangement of pseudolines and let $\overrightarrow{\mathcal{B}}$ be an arrangement which is obtained by a triangular flip from $\overrightarrow{\mathcal{A}}$. We assume that for $\overrightarrow{\mathcal{A}}$ and for all arrangements with fewer lines than $\overrightarrow{\mathcal{A}}$ the claimed inequality holds, i.e., $\operatorname{Min} \operatorname{Inf}(\overrightarrow{\mathcal{A}}) \leq 2 \cdot \operatorname{MaxDis}(\overrightarrow{\mathcal{A}})$. On the basis of this hypothesis we will show that the inequality holds for $\overrightarrow{\mathcal{B}}$.

Let $c$ be the triangular cell in $\overrightarrow{\mathcal{A}}$ which is flipped to get $\overrightarrow{\mathcal{B}}$ and let $T$ be the triple of pseudolines bounding $c$. We distinguish three cases:

- The subsystem induced by $T$ is feasible, i.e., $c$ is on the good side of all three pseudolines.

- The subsystem induced by $T$ is infeasible, i.e., $c$ is on the bad side of all three pseudolines.

- The subsystem induced by $T$ is neither feasible nor infeasible, then we call it neutral.

We first consider the case that subsystem induced by $T$ is neutral. The flip is either left to right or right to left in Figure 13, in either case after performing the flip the subsystem remains neutral. Since $\operatorname{Min} \operatorname{Inf}(\overrightarrow{\mathcal{A}})$ and $\operatorname{MinInf}(\overrightarrow{\mathcal{B}})$ are both at most $i-2$, this value is not affected by the flip. Moreover, a subsystem of $\overrightarrow{\mathcal{A}}$ is infeasible iff it is infeasible in $\overrightarrow{\mathcal{B}}$. Therefore, $\operatorname{MaxDis}(\overrightarrow{\mathcal{A}})=\operatorname{MaxDis}(\overrightarrow{\mathcal{B}})$ and the inequality $\operatorname{Min} \operatorname{Inf}(\overrightarrow{\mathcal{B}}) \leq 2 \cdot \operatorname{MaxDis}(\overrightarrow{\mathcal{B}})$ follows from the corresponding inequality for $\overrightarrow{\mathcal{A}}$.

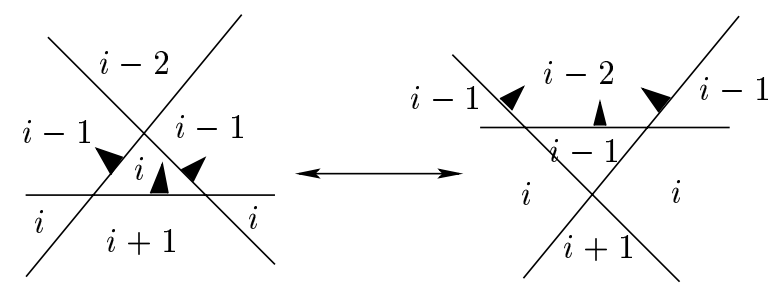

Figure 13: A neutral flip.

Next suppose that the subsystem induced by $T$ is feasible. Let $\overrightarrow{\mathcal{B}}^{*}$ be the arrangement obtained from $\overrightarrow{\mathcal{B}}$ by deleting the three pseudolines of $T$. Recall that by induction

$$
\operatorname{Min} \operatorname{InF}\left(\overrightarrow{\mathcal{B}}^{*}\right) \leq 2 \cdot \operatorname{MAxDis}\left(\overrightarrow{\mathcal{B}}^{*}\right) .
$$


Since $T$ is an infeasible base system of $\overrightarrow{\mathcal{B}}$ we have

$$
\operatorname{MaxDis}\left(\overrightarrow{\mathcal{B}}^{*}\right)+1 \leq \operatorname{MaxDis}(\overrightarrow{\mathcal{B}}) .
$$

To relate the minimum infeasibility of $\overrightarrow{\mathcal{B}}$ and $\overrightarrow{\mathcal{B}}^{*}$ consider a cell $c_{\min }$ of $\overrightarrow{\mathcal{B}}$ with $\operatorname{InF}\left(c_{\text {min }}\right)=\operatorname{MinInF}(\overrightarrow{\mathcal{B}})$. Since $c_{\text {min }} \neq c$ the infeasibility of all points in $c_{\text {min }}$ can decrease by at most two upon removal of the three lines of $T$. Therefore,

$$
\operatorname{MinInf}(\overrightarrow{\mathcal{B}}) \leq \operatorname{MinInF}\left(\overrightarrow{\mathcal{B}}^{*}\right)+2 .
$$

Combining the inequalities leads to the desired result for $\overrightarrow{\mathcal{B}}$ :

$$
\operatorname{Min} \operatorname{Inf}(\overrightarrow{\mathcal{B}}) \leq \operatorname{Min} \operatorname{Inf}\left(\overrightarrow{\mathcal{B}}^{*}\right)+2 \leq 2 \cdot \operatorname{MaxDis}\left(\overrightarrow{\mathcal{B}}^{*}\right)+2 \leq 2 \cdot \operatorname{MaxDis}(\overrightarrow{\mathcal{B}}) .
$$

The last case is that the subsystem induced by $T$ is infeasible. After the flip the subsystem becomes a feasible subsystem of $\overrightarrow{\mathcal{B}}$. Clearly $\operatorname{Min} \operatorname{Inf}(\overrightarrow{\mathcal{A}}) \geq \operatorname{MinInf}(\overrightarrow{\mathcal{B}})$. If $\operatorname{MaxDis}(\overrightarrow{\mathcal{A}})=\operatorname{MaxDis}(\overrightarrow{\mathcal{B}})$ we immediately obtain the result:

$$
\operatorname{Mininf}(\overrightarrow{\mathcal{B}}) \leq \operatorname{MinInf}(\overrightarrow{\mathcal{A}}) \leq 2 \cdot \operatorname{MaxDis}(\overrightarrow{\mathcal{A}})=2 \cdot \operatorname{MaxDis}(\overrightarrow{\mathcal{B}})
$$

The more complex situation is when $\operatorname{MaxDis}(\overrightarrow{\mathcal{A}})-1=\operatorname{MaxDis}(\overrightarrow{\mathcal{B}})$. In this case the three lines of $T$ form an infeasible subsystem of $\overrightarrow{\mathcal{A}}$ which is contained in every maximal set of disjoint infeasible subsystems of $\overrightarrow{\mathcal{A}}$. To deal with this situation we need the following lemma:

Lemma 6. Let $\overrightarrow{\mathcal{A}}$ be an oriented arrangement of pseudolines with an infeasible triangular cell $c$ whose boundary lines form an infeasible system $T$ which is contained in every maximal set of disjoint infeasible subsystems of $\overrightarrow{\mathcal{A}}$. Then

(1) every pseudoline $g$ with $c$ on the bad side is contained in every maximal set of disjoint infeasible subsystems of $\overrightarrow{\mathcal{A}}$, and

(2) there is no infeasible base system $R$ disjoint from $T$ in $\overrightarrow{\mathcal{A}}$ such that $c$ is contained in the bounded region of $R$.

Proof. Let $l_{1}, l_{2}, l_{3}$ be the pseudolines of the system $T$. Suppose there is a pseudoline $g$ which has $c$ on the bad side and is not used by some maximal system of disjoint infeasible subsystems. Up to relabeling the situation is as shown in Figure 14 and replacing the triple $T=l_{1}, l_{2}, l_{3}$ by $g, l_{2}, l_{3}$ gives a maximal system of disjoint infeasible subsystems not containing $T$, a contradiction. 


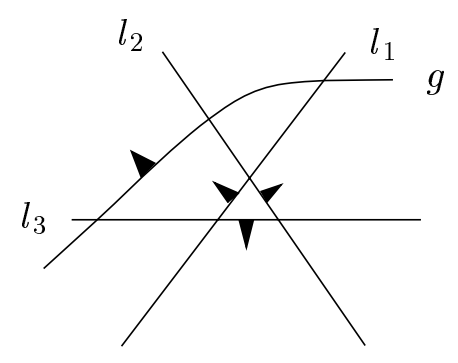

Figure 14: The infeasible triangular cell $c$ and an extra line $g$ with $c$ on its bad side.

For the proof of part 2, suppose there is an infeasible base subarrangement $R$ containing $c$ in its bounded region. Let $g_{1}, g_{2}, g_{3}$ be the pseudolines of $R$. We may assume that the arrangement induced by $l_{1}, l_{2}, l_{3}$ and $g_{1}$ is again as shown in Figure 14.

Suppose that the crossing of $g_{2}$ and $g_{3}$ is on the bad side of $l_{1}$. In this case the triples $l_{2}, l_{3}, g_{1}$ and $g_{2}, g_{3}, l_{1}$ form two disjoint infeasible subarrangements from the six lines of $T$ and $R$.

If $g_{2}$ and $g_{3}$ cross on the good side of $l_{1}$ then the situation is as described by Figure 15 .

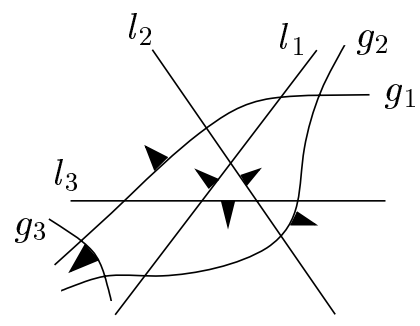

Figure 15: Lines $g_{1}$ and $g_{2}$ cross on the good side of $l_{1}$.

Suppose that the crossing of $l_{2}$ and $g_{3}$ is on the good side of $l_{1}$ and hence on the bad side of $l_{3}$. In this case the triples $l_{2}, l_{3}, g_{3}$ and $l_{1}, g_{1}, g_{2}$ form two disjoint infeasible subarrangements. Otherwise, the crossing of $l_{2}$ and $g_{3}$ is on the bad side of $l_{1}$. In this case the triples $l_{1}, l_{2}, g_{3}$ and $l_{3}, g_{1}, g_{2}$ form two disjoint infeasible subarrangements.

In all cases we can form two new infeasible base arrangements from the six lines of $T$ and $R$. This contradicts the assumption that $T$ is contained in every maximal set of disjoint infeasible subsystems of $\overrightarrow{\mathcal{A}}$.

We now estimate the infeasibility of $c$ in $\overrightarrow{\mathcal{A}}$. Let $F$ be a maximal system 
of disjoint infeasible subsystems in $\overrightarrow{\mathcal{A}}$. Because of Lemma 6.2 . every subarrangement $T^{\prime} \neq T$ in $F$ contains $c$ in a region of infeasibility at most 2 . By Lemma 6.1. this accounts for all pseudolines having $c$ on the bad side. Therefore,

$$
\operatorname{INF}(c) \leq 2 \cdot(\operatorname{MaxDis}(\overrightarrow{\mathcal{A}})-1)+3 \leq 2 \cdot \operatorname{MaxDis}(\overrightarrow{\mathcal{A}})+1 .
$$

Considering the infeasibility of cells in the neighborhood of $c$ we note that $\operatorname{Min} \operatorname{Inf}(\overrightarrow{\mathcal{A}}) \leq \operatorname{InF}(c)-2$. Therefore,

$$
\operatorname{MinInf}(\overrightarrow{\mathcal{A}}) \leq 2 \cdot \operatorname{MaxDis}(\overrightarrow{\mathcal{A}})-1 .
$$

The proof for the case where $\operatorname{MaxDis}(\overrightarrow{\mathcal{B}})=\operatorname{MaxDis}(\overrightarrow{\mathcal{A}})-1$ can be completed using this last inequality. Explicitly,

$$
\operatorname{Min} \operatorname{Inf}(\overrightarrow{\mathcal{B}}) \leq \operatorname{MinInf}(\overrightarrow{\mathcal{A}}) \leq 2 \cdot \operatorname{MaxDis}(\overrightarrow{\mathcal{A}})-1=2 \cdot \operatorname{MaxDis}(\overrightarrow{\mathcal{B}}) .
$$

\section{Conclusion}

We have investigated bounds of the LHS over the RHS in the inequality

$$
\operatorname{MinInf}(\overrightarrow{\mathcal{A}}) \geq \operatorname{MaxDis}(\overrightarrow{\mathcal{A}})
$$

For the 2-dimensional case we gave tight results even for the case of arrangements of pseudolines. In $d$ dimensions we know that the best possible factor is between $d / 2$ and $d$. It would be interesting to know on which side of this interval the truth hides.

\section{References}

[1] S. Felsner AND K. KRIEGeL, Triangles in Euclidean arrangements, Discrete Comput. Geom., 22 (1999), pp. 429-438.

[2] S. Felsner And H. WeIL, A theorem on higher Bruhat orders, Discrete Comput. Geom., 23 (2000), pp. 121-127.

[3] S. FElsner AND H. WeIL, Sweeps, arrangements and signotopes, Discrete Applied Mathematics, (2001). To appear.

[4] S. Felsner AND G. ZIEGLeR, Zonotopes associated with higher bruhat orders, Discrete Mathematics, (2001). to appear in Tverberg Festschrift. 
[5] A. Frank, How to make a digraph strongly connected., Combinatorica, 1 (1981), pp. 145-153.

[6] J. E. Goodman, Pseudoline arrangements, in Handbook of Discrete and Computational Geometry, Goodman and O'Rourke, eds., CRC Press, 1997, pp. 83-109.

[7] M. Grötschel, M. JÜnger, And G. Reinelt, Acyclic subdigraphs and linear orderings, in Graphs and Order, Rival, ed., NATO ASI Ser., Ser. C 147, Reidel, 1985, pp. 217-264.

[8] B. Grünbaum, Arrangements and spreads, Regional Conf. Ser. Math., Amer. Math. Soc., 1972.

[9] L. LovÁsz, On two minimax theorems in graph., J. Comb. Theory, Ser. B, 21 (1976), pp. 96-103.

[10] C. Lucchesi And D. Younger, A minimax theorem for directed graphs., J. London Math. Soc., II. Ser., 17 (1978), pp. 369-374.

[11] Y. Manin And V. Schechtman, Arrangements of hyperplanes, higher braid groups and higher Bruhat orders, in Algebraic Number Theory in honour of K. Iwasawa, J. C. et al., ed., vol. 17 of Advanced Studies in Pure Mathematics, Kinokuniya Company/Academic Press, 1989, pp. 289-308.

[12] G. ZIEGLER, Higher Bruhat orders and cyclic hyperplane arrangements, Topology, 32 (1993), pp. 259-279. 